\title{
The First Report of a Methicillin-Resistant Staphylococcus aureus Isolate Harboring Type IV SCCmec in Thailand
}

\author{
Wichai Santimaleeworagun ${ }^{1,2, *(\mathbb{D})}$, Praewdow Preechachuawong ${ }^{3}$, Wandee Samret ${ }^{4}$ and Tossawan Jitwasinkul ${ }^{5,6}$ \\ 1 Department of Pharmacy, Faculty of Pharmacy, Silpakorn University, Nakhon Pathom 73000, Thailand \\ 2 Pharmaceutical Initiative for Resistant Bacteria and Infectious Diseases Working Group [PIRBIG], \\ Faculty of Pharmacy, Silpakorn University, Nakhon Pathom 73000, Thailand \\ 3 Microbiology Unit, Hua Hin Hospital, Prachuab Kiri Khan 77110, Thailand; santimaleeworag_w@su.ac.th \\ 4 Pharmacy Unit, Hua Hin Hospital, Prachuab Kiri Khan 77110, Thailand; wandeehuahin@gmail.com \\ 5 Department of Biopharmacy, Faculty of Pharmacy, Silpakorn University, Nakhon Pathom 73000, Thailand; \\ tosskiji@gmail.com \\ 6 Bioactives from Natural Resources Research Collaboration for Excellence in Pharmaceutical Sciences (BNEP), \\ Faculty of Pharmacy, Silpakorn University, Nakhon Pathom 73000, Thailand \\ * Correspondence: swichai1234@gmail.com; Tel.:+66-34-255-800; Fax: +66-34-255-801
}

check for

updates

Citation: Santimaleeworagun, W.; Preechachuawong, P.; Samret, W.; Jitwasinkul, T. The First Report of a Methicillin-Resistant Staphylococcus aureus Isolate Harboring Type IV SCCmec in Thailand. Pathogens 2021, 10, 430. https://doi.org/10.3390/ pathogens 10040430

Academic Editor: Valentina

Virginia Ebani

Received: 22 February 2021

Accepted: 1 April 2021

Published: 4 April 2021

Publisher's Note: MDPI stays neutral with regard to jurisdictional claims in published maps and institutional affiliations.

Copyright: (c) 2021 by the authors. Licensee MDPI, Basel, Switzerland. This article is an open access article distributed under the terms and conditions of the Creative Commons Attribution (CC BY) license (https:// creativecommons.org/licenses/by/ $4.0 /)$.
Abstract: Methicillin-resistant Staphylococcus aureus (MRSA) is mostly found in Thailand in the hospital as a nosocomial pathogen. This study aimed to report the genetic characterization of a clinical community-acquired MRSA (CA-MRSA) isolate collected from hospitalized patients in Thailand. Among 26 MRSA isolates, S. aureus no. S17 preliminarily displayed the presence of a staphylococcal cassette chromosome mec (SCCmec) type IV pattern. The bacterial genomic DNA was subjected to whole-genome sequencing. Panton-Valentine leukocidin (PVL) production, virulence toxins, and antibiotic resistance genes were identified, and multi-locus sequence typing (MLST) and spa typing were performed. The strain was matched by sequence to MLST type 2885 and spa type t13880. This strain carried type IV SCCmec with no PVL production. Five acquired antimicrobial resistance genes, namely $\operatorname{blaZ}, \operatorname{mec} A, \operatorname{Inu}(A)$, tet $(K)$, and $\operatorname{dfr} G$ conferring resistance to $\beta$-lactams, lincosamides, tetracycline, and trimethoprim, were identified. The detected toxins were exfoliative toxin A, gamma-hemolysin, leukocidin D, and leukocidin E. Moreover, there were differences in seven regions in CR-MRSA no. S17 compared to CA-MRSA type 300. In summary, we have reported the ST2885-SCCmec IV CA-MRSA clinical strain in Thailand for the first time, highlighting the problem of methicillin resistance in community settings and the consideration in choosing appropriate antibiotic therapy.

Keywords: CA-MRSA; S. aureus; mec IV; whole-genome sequencing

\section{Introduction}

Staphylococcus aureus is presently one of the most important Gram-positive bacteria causing skin/soft tissue, bloodstream, bone/joint infections, pneumonia and endocarditis, in addition to being a rare cause of urinary tract infection and oral infection, in both community and healthcare settings [1-4]. Since the introduction of $\beta$-lactam antibiotics, methicillin-resistant Staphylococcus aureus (MRSA) isolates have been identified. MRSA infection increases the risk of death, the duration of hospitalization, and medical costs $[5,6]$.

$S$. aureus becomes resistant to methicillin after acquiring mec $A$, which is usually carried on the staphylococcal cassette chromosome mec (SCCmec) as a mobile genetic element. $m e c A$ can produce a variant of penicillin-binding protein 2 , which alters the affinity for $\beta$-lactam antibiotics. At present, 13 SCCmec elements have been identified. Of these, SCCmec types IV and V are carried by CA-MRSA strains [7].

MRSA strains are usually the causes of hospital-acquired MRSA (HA-MRSA) infections; however, MRSA infections in the community (community-acquired MRSA (CAMRSA)) have been increasingly reported [8]. Regarding the prevalence of CA-MRSA in 
previous reports, most MRSA infections in Thailand were hospital-associated, whereas CA-MRSA infection was not evident. Only Lulitanond et al. characterized 14 MRSA isolates from infectious outpatients as community onset. Almost all MRSA isolates carried SCCmec type III, whereas only one isolate belonged to SCCmec type IX. Mekviwattanawong et al. suspected CA-MRSA infection in two patients presenting with three MRSA isolates within $72 \mathrm{~h}$ after hospitalization. Unfortunately, the molecular type of these isolates of MRSA was not determined [9]. The characteristics of CA-MRSA strains were not fully investigated, and the reports of the SCCmec types of MRSA are limited. In this study, we performed the first genetic characterization of a clinical CA-MRSA isolate collected from patients in Thailand.

\section{Materials and Methods}

\subsection{Bacterial Isolate}

In our previous studies, we collected 26 MRSA isolates from the clinical specimens of inpatients admitted to Hua Hin Hospital, a 400-bed general hospital in western Thailand, between January 2015 and December 2016 [10]. In our previous findings, three different spa types were identified among 16 isolates, and the proportion of strains assigned to spa type $\mathrm{t} 045$ was $75 \%$. The remaining spa types were $\mathrm{t} 439$ (18.7\%) and $\mathrm{t} 13880(6.3 \%)$. A S. aureus strain S17 isolate belonging to spa type $\mathrm{t} 13880$ exhibited a SCCmec type IV pattern. This isolate was stored in trypticase soy broth plus $15 \%$ glycerol at $-80{ }^{\circ} \mathrm{C}$ for future molecular characterization and antimicrobial susceptibility testing.

\subsection{Phenotypic Study of Antimicrobial Susceptibility Testing}

S. aureus strain S17 (CA-MRSA no. 17) was phenotypically confirmed to be resistant to cefoxitin using the Kirby-Bauer method based on Clinical and Laboratory Standards Institute (CLSI) guidelines [11]. CA-MRSA no. 17 was subjected to antimicrobial susceptibility testing for vancomycin, linezolid, clindamycin, fusidic acid, erythromycin, and trimethoprim/sulfamethoxazole using E-test methods (Liofilchem, Teramo, Italy). The broth microdilution method was based on CLSI guidelines [11].

\subsection{The SCCmec Pattern}

The genomic DNA of CA-MRSA no. 17 isolate was extracted using a commercial kit (Thermo Fisher Scientific, Waltham, MA, USA) with the inclusion of lysostaphin (SigmaAldrich, St. Louis, MO, USA).

For multiplex PCR to determine the SCCmec type, the SCCmec M-PCR typing assay contained nine pairs of primers including the specific primers for SCCmec subtypes I, II, III, IVa, IVb, IVc, IVd, and V and the primers for mecA. The PCR conditions followed previously described protocols [12]. The PCR amplicons were visualized using a UV light box after electrophoresis on a $2 \%$ agarose gel.

\subsection{Whole-Genome Sequencing and Data Analysis}

The representative MRSA isolate was further subjected to whole-genome sequencing. Bacterial genomic DNA was sequenced using the Illumina Miseq platform. The raw whole genome was de novo assembled using the Assembler 1.2 program (https:/ / cge.cbs.dtu. $\mathrm{dk}$ /services / Assembler / accessed on 14 November 2020) [13]. We confirmed bacterial species by submitting the whole-genome sequence to KmerFinder version 3.0.2 (https: / / cge.cbs.dtu.dk/services/KmerFinder/history.php accessed on 14 November 2020) [14].

In the present study, the representative isolates were further classified by clonal relationship based on multi-locus sequence typing (MLST) and then analyzed using MLST 2.0 version 2.0.4 (https: / / cge.cbs.dtu.dk/services/MLST/ accessed on 14 November 2020) [13] and spa typing via spaTyper version 1.0 (https:/ / cge.cbs.dtu.dk/services/spatyper/ accessed on 14 November 2020) [15]. The presence of Panton-Valentine leukocidin (lukF and $l u k S$ ) as a virulence toxin was also analyzed using VirulenceFinder-2.0 (https:/ / cge.cbs. dtu.dk/services/VirulenceFinder/ accessed on 14 November 2020). 
The antibiotic resistance genes obtained via chromosomal mutation and acquired resistance were identified by the Center of Genomic Epidemiology via ResFinder 3.2 (https: / / cge.cbs.dtu.dk/services/ResFinder/ accessed on 14 November 2020). The target genes assessed for chromosomal point mutations included pbp4 (high-level and broadspectrum resistance to the entire class of $\beta$-lactam drugs), fus $A$ (high-level resistance to fusidic acid), gyrA (encoding DNA gyrase for fluoroquinolone resistance), $g r l A / g r l B$ (encoding topoisomerase IV for fluoroquinolone resistance), ileS (conferring mupirocin resistance), $d f r B$ (trimethoprim resistance), $r p o B$ (conferring resistance to rifampin), and $23 S$ (23S rRNA subunit for linezolid resistance). In $S$. aureus, acquired resistance genes included genes related to aminoglycoside, $\beta$-lactam, oxazolidinone, quinolone, macrolide, sulfonamide, fusidic acid, glycopeptide, rifampicin, colistin, fosfomycin, trimethoprim, and tetracycline resistance [16].

The CGView Server Stothard Research Group is a comparative genomics tool for circular genomes that allows sequence feature information to be visualized in the context of sequence analysis results [17].

\subsection{Genome Sequence Data in Sequence Read Archive (SRA)}

The raw reads and assembled contigs of CA-MRSA no. 17 was deposited in SRA (NCBI) under the BioProject ID: PRJNA692064 and BioSample accession number SAMN17313309.

\section{Results}

Phenotypically, the minimum inhibitory concentrations for vancomycin, linezolid, clindamycin, fusidic acid, erythromycin, doxycycline, and trimethoprim/sulfamethoxazole (TMP-SMX) in the strain were $0.38,1.5,0.19,0.13,0.25,8$, and $0.38 \mu \mathrm{g} / \mathrm{mL}$, respectively.

Our studied CA-MRSA no. 17 isolate was confirmed to be $S$. aureus by submitting the whole-genome sequence to KmerFinder. In MLST analysis, this strain was matched to sequence type 2885 . We also confirmed the spa type as t13880 via spaTyper version 1.0. In multiplex PCR assay, CA-MRSA no. S17 carried type IV SCCmec. The strain was confirmed to lack PVL.

We used the ResFinder 3.2 online tool to identify antibiotic resistance genes in our whole-genome assembly. There was a wide distribution of antibiotic resistance genes consisting of $b l a Z / \operatorname{mec} A, \operatorname{Inu}(A)$, tet $(K)$, and $d f r G$ conferring resistance to $\beta$-lactams, macrolides, tetracycline, and TMP, respectively (Table 1 ). There were no mutations found in $p b p 4$, fus $A, g y r A, g r l A, g r l B$, ileS, $d f r B, r p o B$, and 23S. Overall, the antibiotic resistance genotypes exhibited concordance with the observed phenotypic susceptibility testing data. Concerning the presence of toxin genes analyzed via VirulenceFinder-2.0, exfoliative toxin A, gamma-hemolysin, leukocidin D, and leukocidin E were identified (Table 2).

Table 1. The identified antibiotic resistance genes in community acquired methicillin-resistant Staphylococcus aureus (CAMRSA) no. S17.

\begin{tabular}{|c|c|c|c|c|c|c|}
\hline $\begin{array}{l}\text { Resistance } \\
\text { Gene }\end{array}$ & Identity & $\begin{array}{l}\text { Template } \\
\text { Length }\end{array}$ & Contig & $\begin{array}{l}\text { Position in } \\
\text { Contig }\end{array}$ & Predicted Phenotype & $\begin{array}{l}\text { Accession } \\
\text { Number }\end{array}$ \\
\hline & & & $\beta$-lactam & & & \\
\hline blaZ & 100 & $849 / 849$ & NODE_15_length_14257_cov_356.776825 & 7028-7876 & $\beta$-lactam resistance & JBTH01000015 \\
\hline mecA & 100 & $2007 / 2007$ & NODE_1_length_202378_cov_183.284088 & $83,894-85,900$ & $\beta$-lactam resistance & BX571856 \\
\hline $\operatorname{Inu}(\mathrm{A})$ & 99.79 & $486 / 486$ & $\begin{array}{c}\text { Macrolide } \\
\text { NODE_34_length_2491_cov_187.230835 }\end{array}$ & $2007-2492$ & Lincosamide resistance & M14039 \\
\hline tet(K) & 99.86 & $1380 / 1380$ & $\begin{array}{c}\text { Tetracycline } \\
\text { NODE_6_length_4546_cov_373.607574 }\end{array}$ & $2335-3714$ & Tetracycline resistance & U38656 \\
\hline dfrG & 100 & $498 / 498$ & $\begin{array}{c}\text { Trimethoprim } \\
\text { NODE_1_length_202378_cov_183.284088 }\end{array}$ & $90,645-91,142$ & Trimethoprim resistance & AB205645 \\
\hline
\end{tabular}


Table 2. The identified genes of virulence factor in CA-MRSA no. S17.

\begin{tabular}{ccccccc}
\hline $\begin{array}{c}\text { Virulence } \\
\text { Factor }\end{array}$ & Identity & $\begin{array}{c}\text { Template } \\
\text { Length }\end{array}$ & \multicolumn{1}{c}{ Contig } & $\begin{array}{c}\text { Position in } \\
\text { Contig }\end{array}$ & $\begin{array}{c}\text { Protein Function } \\
\text { Number }\end{array}$ \\
\hline eta & 100 & $843 / 843$ & NODE_140_length_521104_cov_145.043243 & 517966.518808 & Exfoliative toxin A & AP008953.1 \\
\hline$h l g A$ & 99.89 & $930 / 930$ & NODE_83_length_193242_cov_168.671219 & 110976.111905 & $\begin{array}{c}\text { Gamma-hemolysin chain II } \\
\text { precursor }\end{array}$ \\
\hline$h l g B$ & 100 & $977 / 977$ & NODE_83_length_193242_cov_168.671219 & $113422 . .114398$ & $\begin{array}{c}\text { Gamma-hemolysin } \\
\text { component B precursor }\end{array}$ AP01494242.1 \\
\hline$h l g C$ & 99.79 & $948 / 948$ & NODE_83_length_193242_cov_168.671219 & $112473 . .113420$ & $\begin{array}{c}\text { Gamma-hemolysin } \\
\text { component C }\end{array}$ \\
\hline$l u k D$ & 99.8 & $984 / 984$ & NODE_118_length_478021_cov_147.302170 & $469250 . .470233$ & Leukocidin D component & AP014653.1 \\
\hline$l u k E$ & 99.89 & $936 / 936$ & NODE_118_length_478021_cov_147.302170 & $470235 . .471170$ & Leukocidin E component & BA000018.3 \\
\hline$l u k E$ & 99.89 & $936 / 936$ & NODE_118_length_478021_cov_147.302170 & 470235.471170 & Leukocidin E component & CP001781.1 \\
\hline
\end{tabular}

We compared the circular genome sequence $(2,904,778$ base pairs) of the studied strain to that of CA-MRSA type USA300, finding differences in seven regions (Figure 1).

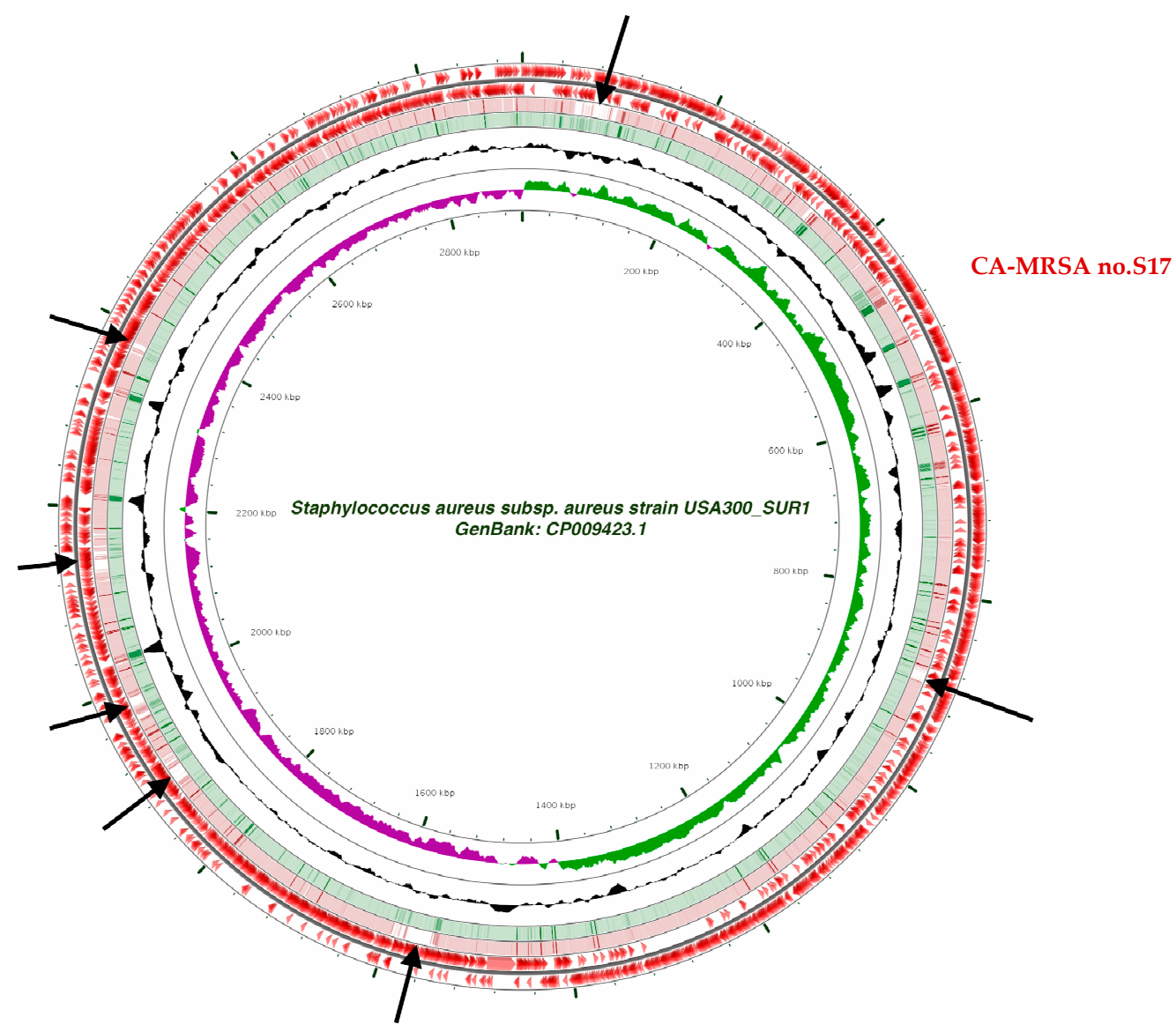

Figure 1. CA-MRSA no. S17 circular genome map (2,904,778 base pairs) compared to CA-MRSA type USA300. Black arrows denote the positions of sequence differences between CA-MRSA type USA300 and CA-MRSA no. S17.

\section{Discussion}

MRSA was initially recognized as a nosocomial pathogen. The bacterium was not isolated in the community setting until 1961. However, the first definite case of CA-MRSA was reported in 1993 in Western Australia. Subsequently, CA-MRSA was identified in the United States in children who died of sepsis or necrotizing pneumonia between 1997 and 1999. Currently, CA-MRSA strain USA300 is a major cause of CA-MRSA infections in the United States and Canada [7]. 
As previously described, CA-MRSA has been particularly recognized in the United States over the last two decades. The burden of CA-MRSA infection has shifted the empirical antimicrobial therapy for skin and soft-tissue infection [18]. The United States Centers for Disease Control and Prevention gave the standard definition in 2005 that a CA-MRSA infection is any MRSA infection diagnosed in an outpatient or patient admitted to a hospital within the preceding $48 \mathrm{~h}$ [7]. Otherwise, MRSA infections are considered to be HA-MRSA. Our CA-MRSA strain was isolated from a hospitalized patient. However, HA-MRSA strains are also present in the community, especially in patients who required acute care hospital for $\geq 2$ days within the previous 90 days, whereas CA-MRSA isolates can be transmissible in the hospital [18]. Nosocomial transmission and hospital outbreaks of CA-MRSA have been reported in certain countries $[19,20]$. Thus, the CA-MRSA isolate obtained in this report is the first reported strain in Thailand despite being isolated from a hospitalized patient.

PVL, a cytotoxic toxin, is associated with severe pneumonia and skin/soft-tissue infections caused by $S$. aureus. PVL is encoded by lukS-PV and lukF-PV. PVL mainly destroys polymorphonuclear leukocytes, monocytes, and macrophages, leading to cell destruction and the release of pro-inflammatory cytokines and nuclear factor-kappa B [21]. PVL production results in necrotizing damage such as lung injury and deep-seated skin and soft-tissue infections [22,23]. PVL-positive S. aureus is present in most patients with community-acquired necrotizing pneumonia, among whom the mortality rate ranges between $40 \%-60 \%$ [24].

The prevalence of PVL-positive S. aureus among methicillin-resistant isolates varies. Moran et al. enrolled adult patients with purulent skin and soft-tissue infections across 11 university-affiliated emergency departments in the United States in 2004. Of 214 MRSA isolates characterized as SCCmec type IV, pvl was present in 213 isolates [25]. Similarly, Nakaminami et al. found that all CA-MRSA isolates with SCCmec type IV were PVLpositive [26]. However, in other studies, the prevalence of PVL-positive CA-MRSA was the same as that of PVL-negative CA-MRSA $[27,28]$. Thus, the absence of $p v l$ in our CA-MRSA isolate might be reasonable.

Although our CA-MRSA isolate lacked $l u k S-P V$ and $l u k F-P V$, other virulence factors such as exfoliative toxin, hemolysin, and leukocidin were present. Our results support that CA-MRSA strains usually produce hemolysins, leukocidin, or exfoliative toxins, whereas HA-MRSA usually does not produce such toxins. Moreover, CA-MRSA strains also produce $\beta$-lactamase and hyaluronidase [7], consistent with the findings for our CA-MRSA isolate.

Unlike SCCmec types I-III, which carry multiple genes for various antibiotic resistance, SCCmec type IV only carries mecA conferring resistance to $\beta$-lactams. CA-MRSA strains are usually susceptible to tetracyclines, clindamycin, and TMP-SMX but not $\beta$-lactams [7]. These susceptibility patterns were also found in our CA-MRSA isolate. Thus, clindamycin, and TMP-SMX might be options for skin and soft-tissue infections. For complicated skin and soft-tissue infections and invasive MRSA infections caused by CA-MRSA, such as bloodstream infections, endocarditis, meningitis, and pneumonia, parenteral vancomycin is the recommended treatment. Linezolid might be an alternative agent for severe MRSA [29].

To our knowledge, this is the first report of a CA-MRSA isolate (spa type t13880) carrying SCCmec IV from patients in Thailand. Unfortunately, HA-MRSA strains have been reported in Thailand, most commonly ST239-SCCmec III type followed by ST5-SCCmec II $[30,31]$. CA-MRSA infection has been rarely documented. Only a study by Lulitanond et al. in 2013 isolated two MRSA strains (one each from pig farm workers and a pig) of ST9-SCCmec IX in the community [32]. SCCmec IX MRSA (belonging to spa type t337) was also reported by Larsen et al. in pigs in Thailand [33]. Thus, the emergence of SCCmec IX in animal farms in the community is concerning.

The reported CA-MRSA clones vary by region/country, including ST1-SCCmec IV (USA400) and ST8-SCCmec IV strains (USA300) in the United States, ST30-SCCmec IV and ST93-SCCmec IV strains in Australia, ST22-SCCmec IV strains in India, and CC80-SCCmec IV strains in Europe and Africa [34]. 
Interestingly, we classified our CA-MRSA isolate as spa type t13880 belonging to ST2885 by MLST analysis. This MRSA-ST288517 clone had only been identified in the Lao People's Democratic Republic (bordering country of Thailand), but the ST2885 isolates belonged to spa type t13849. Additionally, these ST2885 isolates carried eta (encoding exfoliative toxin A), which was also found in our studied CA-MRSA isolate. However, most ST2885 strains are methicillin-sensitive S. aureus [35]. Therefore, the present study confirmed the existence of the ST2885 clone in Southeast Asia, albeit with a different methicillin susceptibility pattern.

Previously, Mekviwattanawong et al. determined the prevalence of infections caused by CA-MRSA among hospitalized patients, with MRSA isolated from the patients within $72 \mathrm{~h}$ of hospitalization. CA-MRSA was found in 2 of 186 patients with MRSA infection. The infections were likely caused by HA-MRSA because both patients had previous histories of hospital visits prior to their hospitalization. Moreover, the isolates defined as CA-MRSA strains were not subjected to SCCmec typing, and their antibiotic susceptibility profiles indicated multidrug resistance [9]. Thus, the situation of CA-MRSA in Thailand could not be confirmed until the present study. Treatment regimens for infections caused by S. aureus in the community setting should be developed.

\section{Conclusions}

We have isolated the ST2885-SCCmec IV CA-MRSA strain from a patient admitted to the hospital for the first time in Thailand. The presence of this isolate highlights the problem of methicillin-resistant infections in the community setting.

Author Contributions: Conceptualization, W.S. (Wichai Santimaleeworagun); methodology, W.S. (Wichai Santimaleeworagun), T.J.; validation, W.S. (Wichai Santimaleeworagun), T.J.; formal analysis, W.S. (Wichai Santimaleeworagun), T.J.; investigation, W.S. (Wichai Santimaleeworagun), P.P.; resources, W.S. (Wandee Samret), P.P.; data curation, W.S. (Wichai Santimaleeworagun), P.P.; writingoriginal draft preparation, W.S. (Wichai Santimaleeworagun); writing-review and editing, P.P., W.S. (Wandee Samret) and T.J.; supervision, W.S.; project administration, W.S. (Wichai Santimaleeworagun); funding acquisition, W.S. (Wichai Santimaleeworagun). All authors have read and agreed to the published version of the manuscript.

Funding: This research was funded by Faculty of Pharmacy, Silpakorn University.

Institutional Review Board Statement: The clinical specimen was part of the routine hospital laboratory procedure. The use of cultured pathogen from human specimen was approved by the institutional review board of Hua Hin Hospital [COE007/2560; RECHHH043/2560] and was carried out in accordance with the approved guidelines.

Informed Consent Statement: Not applicable.

Data Availability Statement: The data presented in this study are available in article.

Conflicts of Interest: The authors declare no conflict of interest.

\section{References}

1. Purrello, S.M.; Garau, J.; Giamarellos, E.; Mazzei, T.; Pea, F.; Soriano, A.; Stefani, S. Methicillin-resistant Staphylococcus aureus infections: A review of the currently available treatment options. J. Glob. Antimicrob. Resist. 2016, 7, 178-186. [CrossRef] [PubMed]

2. Nickerson, E.K.; West, T.E.; Day, N.P.; Peacock, S.J. Staphylococcus aureus disease and drug resistance in resource-limited countries in south and east Asia. Lancet Infect. Dis. 2009, 9, 130-135. [CrossRef]

3. Tong, S.Y.; Davis, J.S.; Eichenberger, E.; Holland, T.L.; Fowler, V.G., Jr. Staphylococcus aureus infections: Epidemiology, pathophysiology, clinical manifestations, and management. Clin. Microbiol. Rev. 2015, 28, 603-661. [CrossRef] [PubMed]

4. Marrelli, M.; Tatullo, M.; Dipalma, G.; Inchingolo, F. Oral infection by Staphylococcus aureus in patients affected by White Sponge Nevus: A description of two cases occurred in the same family. Int. J. Med. Sci. 2012, 9, 47-50. [CrossRef]

5. Chaiwarith, R.; Pacharasupal, P.; Sirisanthana, T. Epidemiology, clinical characteristics and treatment outcomes of healthcare- associated methicillin-resistant Staphylococcus aureus BLOODSTREAM infections at Chiang Mai University Hospital: A retrospective study. Southeast Asian J. Trop. Med. Public Health 2014, 45, 897-905. 
6. De Kraker, M.E.; Davey, P.G.; Grundmann, H.; Burden Study Group. Mortality and hospital stay associated with resistant Staphylococcus aureus and Escherichia coli bacteremia: Estimating the burden of antibiotic resistance in Europe. PLoS Med. 2011, 8, e1001104. [CrossRef]

7. Lakhundi, S.; Zhang, K. Methicillin-Resistant Staphylococcus aureus: Molecular Characterization, Evolution, and Epidemiology. Clin. Microbiol. Rev. 2018, 31, e00020-18. [CrossRef] [PubMed]

8. Huh, K.; Chung, D.R. Changing epidemiology of community-associated methicillin-resistant Staphylococcus aureus in the AsiaPacific region. Expert Rev. Anti Infect. Ther. 2016, 14, 1007-1022. [CrossRef]

9. Mekviwattanawong, S.; Srifuengfung, S.; Chokepaibulkit, K.; Lohsiriwat, D.; Thamlikitkul, V. Epidemiology of Staphylococcus aureus infections and the prevalence of infection caused by community-acquired methicillin-resistant Staphylococcus aureus in hospitalized patients at Siriraj Hospital. J. Med. Assoc. Thai. 2006, 89 (Suppl. S5), S106-S117.

10. Santimaleeworagun, W.; Jitwasinkul, T.; Preechachuawong, P.; Samret, W. Mono sulfamethoxazole/trimethoprim and vancomycin combination antimicrobial activity against methicillin-resistant Staphylococcus aureus. Southeast Asian J. Trop. Med. Public Health 2020, 51, 115-123.

11. Clinical and Laboratory Standards Institute. Performance Standards for Antimicrobial Susceptibility Testing: Twenty-Fifth Informational Supplement; Clinical and Laboratory Standards Institute: Wayne, PA, USA, 2015; p. 177.

12. Zhang, K.; McClure, J.A.; Elsayed, S.; Louie, T.; Conly, J.M. Novel multiplex PCR assay for characterization and concomitant subtyping of staphylococcal cassette chromosome mec types I to V in methicillin-resistant Staphylococcus aureus. J. Clin. Microbiol. 2005, 43, 5026-5033. [CrossRef] [PubMed]

13. Larsen, M.V.; Cosentino, S.; Rasmussen, S.; Friis, C.; Hasman, H.; Marvig, R.L.; Jelsbak, L.; Sicheritz-Ponten, T.; Ussery, D.W.; Aarestrup, F.M.; et al. Multilocus sequence typing of total-genome-sequenced bacteria. J. Clin. Microbiol. 2012, 50, 1355-1361. [CrossRef] [PubMed]

14. Center for Genomic Epidemiology. Prediction of Bacterial Species Using a Fast K-mer Algorithm. Available online: https: / / cge.cbs.dtu.dk/services/KmerFinder/ (accessed on 14 November 2020).

15. Bartels, M.D.; Petersen, A.; Worning, P.; Nielsen, J.B.; Larner-Svensson, H.; Johansen, H.K.; Andersen, L.P.; Jarlov, J.O.; Boye, K.; Larsen, A.R.; et al. Comparing whole-genome sequencing with Sanger sequencing for spa typing of methicillin-resistant Staphylococcus aureus. J. Clin. Microbiol. 2014, 52, 4305-4308. [CrossRef] [PubMed]

16. Zankari, E.; Hasman, H.; Cosentino, S.; Vestergaard, M.; Rasmussen, S.; Lund, O.; Aarestrup, F.M.; Larsen, M.V. Identification of acquired antimicrobial resistance genes. J. Antimicrob. Chemother. 2012, 67, 2640-2644. [CrossRef]

17. Grant, J.R.; Stothard, P. The CGView Server: A comparative genomics tool for circular genomes. Nucleic Acids Res. 2008, 36, W181-W184. [CrossRef]

18. Khan, A.; Wilson, B.; Gould, I.M. Current and future treatment options for community-associated MRSA infection. Expert Opin. Pharm. 2018, 19, 457-470. [CrossRef]

19. Elston, J.W.; Barlow, G.D. Community-associated MRSA in the United Kingdom. J. Infect. 2009, 59, 149-155. [CrossRef]

20. Park, S.H.; Park, C.; Yoo, J.H.; Choi, S.M.; Choi, J.H.; Shin, H.H.; Lee, D.G.; Lee, S.; Kim, J.; Choi, S.E.; et al. Emergence of community-associated methicillin-resistant Staphylococcus aureus strains as a cause of healthcare-associated bloodstream infections in Korea. Infect. Control Hosp. Epidemiol. 2009, 30, 146-155. [CrossRef]

21. Saeed, K.; Gould, I.; Esposito, S.; Ahmad-Saeed, N.; Ahmed, S.S.; Alp, E.; Bal, A.M.; Bassetti, M.; Bonnet, E.; Chan, M.; et al Corrigendum to 'Panton-Valentine Leucocidin (PVL) Staphylococcus aureus a position statement from the International Society of Chemotherapy' [International Journal of Antimicrobial Agents 51/1 (2018) 16-25]. Int. J. Antimicrob. Agents 2018, 52, 125. [CrossRef]

22. Kreienbuehl, L.; Charbonney, E.; Eggimann, P. Community-acquired necrotizing pneumonia due to methicillin-sensitive Staphylococcus aureus secreting Panton-Valentine leukocidin: A review of case reports. Ann. Intensive Care 2011, 1, 52. [CrossRef]

23. Yamasaki, O.; Kaneko, J.; Morizane, S.; Akiyama, H.; Arata, J.; Narita, S.; Chiba, J.; Kamio, Y.; Iwatsuki, K. The association between Staphylococcus aureus strains carrying panton-valentine leukocidin genes and the development of deep-seated follicular infection. Clin. Infect. Dis. 2005, 40, 381-385. [CrossRef]

24. Lina, G.; Piemont, Y.; Godail-Gamot, F.; Bes, M.; Peter, M.O.; Gauduchon, V.; Vandenesch, F.; Etienne, J. Involvement of PantonValentine leukocidin-producing Staphylococcus aureus in primary skin infections and pneumonia. Clin. Infect. Dis. 1999, 29, 1128-1132. [CrossRef]

25. Moran, G.J.; Krishnadasan, A.; Gorwitz, R.J.; Fosheim, G.E.; McDougal, L.K.; Carey, R.B.; Talan, D.A.; EMERGEncy ID Net Study Group. Methicillin-resistant S. aureus infections among patients in the emergency department. N. Engl. J. Med. 2006, 355, 666-674. [CrossRef] [PubMed]

26. Nakaminami, H.; Ito, A.; Sakanashi, D.; Suematsu, H.; Yamagishi, Y.; Mikamo, H.; Noguchi, N. Genetic diversity of pvl-positive community-onset methicillin-resistant Staphylococcus aureus isolated at a university hospital in Japan. J. Infect. Chemother. 2017, 23, 856-858. [CrossRef]

27. Asiimwe, B.B.; Baldan, R.; Trovato, A.; Cirillo, D.M. Molecular epidemiology of Panton-Valentine Leukocidin-positive communityacquired methicillin resistant Staphylococcus aureus isolates in pastoral communities of rural south western Uganda. BMC Infect. Dis. 2017, 17, 24. [CrossRef] [PubMed]

28. Hewagama, S.; Spelman, T.; Woolley, M.; McLeod, J.; Gordon, D.; Einsiedel, L. The Epidemiology of Staphylococcus aureus and Panton-Valentine Leucocidin (pvl) in Central Australia, 2006-2010. BMC Infect. Dis. 2016, 16, 1-6. [CrossRef] 
29. Loewen, K.; Schreiber, Y.; Kirlew, M.; Bocking, N.; Kelly, L. Community-associated methicillin-resistant Staphylococcus aureus infection: Literature review and clinical update. Can. Fam. Physician 2017, 63, 512-520. [PubMed]

30. Chongtrakool, P.; Ito, T.; Ma, X.X.; Kondo, Y.; Trakulsomboon, S.; Tiensasitorn, C.; Jamklang, M.; Chavalit, T.; Song, J.H.; Hiramatsu, K. Staphylococcal cassette chromosome mec (SCCmec) typing of methicillin-resistant Staphylococcus aureus strains isolated in 11 Asian countries: A proposal for a new nomenclature for SCCmec elements. Antimicrob. Agents Chemother. 2006, 50, 1001-1012. [CrossRef]

31. Lulitanond, A.; Chanawong, A.; Sribenjalux, P.; Wilailuckana, C.; Kaewkes, W.; Vorachit, M.; Ito, T.; Hiramatsu, K. Preliminary report of SCCmec-types and antimicrobial susceptibilities of methicillin-resistant Staphylococcus aureus isolates from a university hospital in Thailand. Southeast. Asian J. Trop Med. Public Health 2010, 41, 920-927.

32. Sinlapasorn, S.; Lulitanond, A.; Angkititrakul, S.; Chanawong, A.; Wilailuckana, C.; Tavichakorntrakool, R.; Chindawong, K.; Seelaget, C.; Krasaesom, M.; Chartchai, S.; et al. SCCmec IX in meticillin-resistant Staphylococcus aureus and meticillin-resistant coagulase-negative staphylococci from pigs and workers at pig farms in Khon Kaen, Thailand. J. Med. Microbiol. 2015, 64, 1087-1093. [CrossRef]

33. Larsen, J.; Imanishi, M.; Hinjoy, S.; Tharavichitkul, P.; Duangsong, K.; Davis, M.F.; Nelson, K.E.; Larsen, A.R.; Skov, R.L. Methicillin-resistant Staphylococcus aureus ST9 in pigs in Thailand. PLoS ONE 2012, 7, e31245. [CrossRef] [PubMed]

34. Witte, W. Community-acquired methicillin-resistant Staphylococcus aureus: What do we need to know? Clin. Microbiol. Infect. 2009, 15 (Suppl. S7), 17-25. [CrossRef] [PubMed]

35. Yeap, A.D.; Woods, K.; Dance, D.A.B.; Pichon, B.; Rattanavong, S.; Davong, V.; Phetsouvanh, R.; Newton, P.N.; Shetty, N.; Kearns, A.M. Molecular Epidemiology of Staphylococcus aureus Skin and Soft Tissue Infections in the Lao People's Democratic Republic. Am. J. Trop. Med. Hyg. 2017, 97, 423-428. [CrossRef] [PubMed] 